\title{
Global existence and asymptotic behavior of solutions to a nonlocal Fisher-KPP type problem
}

\author{
Shen Bian* $\quad$ Li Chen ${ }^{\dagger} \quad$ Evangelos A. Latos ${ }^{\ddagger}$
}

\begin{abstract}
In this work, we consider a nonlocal Fisher-KPP reaction-diffusion problem with Neumann boundary condition and nonnegative initial data in a bounded domain in $\mathbb{R}^{n}(n \geq 1)$, with reaction term $u^{\alpha}(1-m(t))$, where $m(t)$ is the total mass at time $t$. With the help of Pohožaev's identity, the non-existence of nontrivial stationary solutions is being shown. When $\alpha \geq 1$ and the initial mass is greater than or equal to one, the problem has nonnegative classical solutions. While if the initial mass is less than one, then the problem admits global solutions for $n=1,2$ with any $1 \leq \alpha<2$ or $n \geq 3$ with any $1 \leq \alpha<1+2 / n$. Moreover, the asymptotic convergence to the solution of the heat equation is proved.
\end{abstract}

\section{Introduction}

In this work we consider the following nonlocal initial boundary value problem,

$$
\begin{array}{ll}
u_{t}-\Delta u=u^{\alpha}\left(1-\int_{\Omega} u(x, t) d x\right), & x \in \Omega, t>0, \\
\mathcal{B} u=0, & x \in \partial \Omega, \\
u(x, 0)=u_{0}(x) \geq 0, & x \in \Omega,
\end{array}
$$

where $u$ is the density, $\Omega$ is a smooth bounded domain in $\mathbb{R}^{n}, n \geq 1, \alpha \geq 1$ and $\nu$ is the outer unit normal vector on $\partial \Omega$. Here $\mathcal{B}$ describes the boundary condition. Without loss of generality, throughout this paper we assume $|\Omega|=1$ (otherwise, rescale the problem by $|\Omega|$ ), let $m(t)=$

*Beijing University of Chemical Technology, 100029, Beijing. Email: bianshen66@163.com. Partially supported by China Postdoctoral Science Foundation, No. 2014M560037.

${ }^{\dagger}$ Universität Mannheim, 68131, Mannheim. Email: chen@math.uni-mannheim.de. Partially supported by the National Natural Science Foundation of China (NSFC), No. 11271218.

${ }^{\ddagger}$ Universität Mannheim, 68131, Mannheim. Email: evangelos.latos@math.uni-mannheim.de. 
$\int_{\Omega} u(x, t) d x$ and $m_{0}=m(0)$. A damping term with $\sigma>0$ can also be included to get $u_{t}-\Delta u+\sigma u=$ $u^{\alpha}(1-m(t))$. In this case, similar results to this paper can also be obtained. For simplicity, we assume that $\sigma=0$.

In the 1930s, Fisher [10] and Kolmogorov, Petrovskii, Piskunov [17] in population dynamics and Zeldovich, Frank-Kamenetskii [35] in combustion theory started to study problems with this kind of reaction terms. Actually, they introduced the scalar reaction-diffusion equation $\frac{\partial u}{\partial t}=\frac{\partial^{2} u}{\partial x^{2}}+F(u)$, and studied the existence, stability and speed of propagation. In the theory of population dynamics, the function $F$ is considered as the rate of the reproduction of the population. It is usually of the form

$$
F(u)=\beta u^{\alpha}(1-u)-\gamma u
$$

From the above model two cases emerge depending on the values of $\alpha$.

In the case of $\alpha=1$, the reproduction rate is proportional to the density $u$ of the population and to available resources $(1-u)$. The last term, $-\gamma u$, describes the mortality of the population.

The case $\alpha=2$, which is the motivation for our work, considers the addition of sexual reproduction to the model with the reproduction rate proportional to the square of the density, see [31]. For more information on reaction-diffusion waves in biology, we refer to the review paper of Volpert and Petrovskii [30].

Next we pass to the relation between the local and the non-local consumption of resources. In the local reaction-diffusion problem

$$
\frac{\partial u}{\partial t}=\frac{\partial^{2} u}{\partial x^{2}}+\beta u^{\alpha}(1-u)-\gamma u
$$

where $u$ is the population density, $\frac{\partial^{2} u}{\partial x^{2}}$ describes the random displacement of the individuals of this population and the reaction term represents their reproduction and mortality. Moreover, the reaction term consists of the reproduction term which is represented by the population density to a power, $u^{\alpha}$, multiplied with the term $(1-u)$ which stands for the local consumption of available resources.

The nonlocal version of the above problem is

$$
\frac{\partial u}{\partial t}=\frac{\partial^{2} u}{\partial x^{2}}+\beta u^{\alpha}\left(1-\int_{-\infty}^{\infty} \phi(x-y) u(y, t) d y\right)-\gamma u
$$

where $\beta, \gamma>0$ and $\int_{-\infty}^{\infty} \phi(y) d y=1$. It can be seen as the case where the individual, located at a certain point, can consume resources in some area around that point. $\phi(x-y)$ represents the probability density function that describes the distribution of individuals around their average 
positions and it depends on the distance from the average point $x$ to the actual point $y$. One can easily verify that if $\phi$ is a Dirac $\delta$-function, then the nonlocal problem reduces to (2).

In the current paper we will study problems with reaction terms similar to the above nonlocal reaction terms. There are some already known results on the reaction-diffusion equation with a nonlocal term,

$$
u_{t}=\Delta u+F(t, u, I(u)), \quad I(u)=\int_{\Omega} u(y, t) d y,
$$

in a bounded domain $\Omega$. However, compared to the local version, the results for the nonlocal reaction terms of Fisher-KPP type are relatively limited. Here we list some of the known recent results.

Anguiano, Kloeden and Lorenz considered $F=f(u) I\left(u^{4}\right)\left(1-I\left(u^{4}\right)\right)$ and proved the existence of a global attractor [1]. Wang and Wo [33] proved the convergence to a stationary solution with $F=u^{m}-I\left(u^{m}\right), m>1$. For $F=\alpha e^{\gamma u}+b I\left(e^{\gamma u}\right)$, Pao [24] studied the existence or nonexistence of stationary solutions. Liu, Chen and Lu [20] proved also the blow-up of solutions for a similar equation, see also [9]. Rouchon obtained global estimates of solutions in [27]. For more information on nonlocal KPP-Fisher type problems, we refer to a recent book by Volpert [29].

Nonlocal Fisher-KPP type reaction terms can describe also Darwinian evolution of a structured population density or the behavior of cancer cells with therapy as well as polychemotherapy and chemotherapy, we refer the interested reader to the models found in [21, 22, 23].

Bebernes and Bressan [4] (see also Bebernes [5], Pao[24]) considered the equation with reaction term

$$
F(t, u, I(u))=f(t, u(t, x))+\int_{\Omega} g(t, u(t, y)) d y, \quad t>0, \quad x \in \Omega .
$$

They considered the case when $f(t, u)=e^{u}, g(t, u)=k e^{u}(k>0)$, for which the above problem represents an ignition model for a compressible reactive gas, and proved that solutions blow-up.

Later, Wang and Wang [34] considered a power-like nonlinearity, i.e.

$$
F(t, u, I(u))=\int_{\Omega} u^{p}(t, y) d y-k u^{q}(t, x), \quad t>0, \quad x \in \Omega,
$$

with $p, q>1$, and proved the blow-up of the solutions.

Budd, Dold and Stuart [8], Hu and Yin [15] considered a similar to the above problem in the case $p=2$ and general $p$ respectively,

$$
F(t, u, I(u))=u^{p}-\frac{1}{|\Omega|} \int_{\Omega} u^{p}(t, y) d y, \quad t>0, \quad x \in \Omega .
$$

With this typical structure, the energy of the solutions is conserved (under Neumann boundary conditions). For this kind of nonlocal problems it is known [34] that there is no comparison principle 
and they are the closest models to the ones we are considering in this work. For a general study on nonlocal problems, we refer to Quittner's and Souplet's book [25] as well as the paper by Souplet $[26]$.

In this article, we will focus on (1) which has a reaction term of the type

$$
F(t, u, I(u))=u^{\alpha}\left(1-\int_{\Omega} u(x, t) d x\right)
$$

for $\alpha \geq 1$. One additional fact that makes this problem more difficult to handle is the lack of comparison principle as one can see for example from [34].

For nonnegative $u$ and Neumann boundary condition, formally by integrating (1) over $\Omega$, we get,

$$
m^{\prime}(t)=(1-m(t)) \int_{\Omega} u^{\alpha} d x
$$

where $m(t)=\int_{\Omega} u(x, t) d x$ is the total mass at time $t$.

If we start at time $t_{0}$ such that $1-m\left(t_{0}\right)<0$, which means that $m\left(t_{0}\right)>1$, we can see that $m^{\prime}(t)$ is negative and therefore $m(t)$ decreases in time. In this case it is natural to expect the global existence of solutions. On the other hand, if we start at time $t_{0}$ such that $1-m\left(t_{0}\right)>0$, we can see that $m(t)$ increases in time. However if $(1-m(t))$ remains positive, the equation has a similar structure to the heat equation with a power-like reaction term for which we know that the problem might have no global solution (see for example $[2,3,11,12,13,16]$ ). For a more detailed discussion on the possible appearance of singularities, see Section 2.

In this paper we prove the global existence of classical solution and its asymptotic behavior for appropriate $\alpha$. The main results are the following two theorems:

Theorem 1. Let $n \geq 1, \alpha \geq 1$ and $\int_{\Omega} u_{0}(x) d x=m_{0}>0$. Assume $u_{0}$ is nonnegative and $u_{0} \in L^{k}(\Omega)$ for any $1<k<\infty$. Then for $m_{0}<1$ with $\alpha$ satisfying

$$
\begin{aligned}
& 1 \leq \alpha<1+2 / n, n \geq 3, \\
& 1 \leq \alpha<2, \quad n=1,2
\end{aligned}
$$

or $m_{0} \geq 1$ with arbitrary $\alpha \geq 1$, problem (1) with $\mathcal{B} u=\partial_{\nu} u$ has nonnegative classical solutions. Moreover, the following a priori estimates hold true. That's for $m_{0}<1$,

$$
\|u(\cdot, t)\|_{L^{k}(\Omega)} \leq C+C t^{-\frac{k-1}{\alpha-1}} \quad \text { for any } t>0 .
$$

For $m_{0} \geq 1$

$$
\begin{gathered}
\|u\|_{L^{k}(\Omega)}^{k} \leq C+C t^{-\frac{n(k-1)}{2}}, \quad n \geq 3, \\
\|u\|_{L^{k}(\Omega)}^{k} \leq C+C t^{-(k-1)}, \quad n=1,2 .
\end{gathered}
$$


Here $C$ denote different constants depending on $m_{0}, k, \alpha$, but not depending on $\left\|u_{0}\right\|_{L^{k}(\Omega)}$.

Theorem 2. Let $u(x, t)$ be a nonnegative classical solution obtained from Theorem $1, v$ be the solution to the heat equation with Neumann boundary condition and initial data $\int_{\Omega} v_{0}(x) d x=m_{0}$, then,

$$
\left\|u(\cdot, t)-v(\cdot, t)-\left(1-m_{0}\right)\right\|_{L^{2}(\Omega)} \leq C_{1} e^{-C_{2} t},
$$

where $C_{1}, C_{2}$ are constants depending on the initial mass $m_{0}$ and $\left\|u_{0}\right\|_{L^{2 \alpha}(\Omega)}$.

This paper is organized as follows. Section 2 investigates the corresponding steady state problem to (1) and discusses the possibility of blow-up for the time dependent case. In section 3 we begin by presenting the dynamics of the mass and then the global existence of the solutions to (1) for appropriate $\alpha$ and thus the proof the Theorem 1 are shown. The last part of this section is devoted to the proof of Theorem 2.

\section{Non-existence of stationary states and possible singularities}

This section is composed of two parts. The first one talks about the non-existence of stationary states for appropriate $\alpha$ and $m_{0}$ via Pohožaev's method. And, the second part discusses the possibility of blow-up phenomena.

The corresponding stationary problem with Dirichlet boundary condition is the following,

$$
\begin{aligned}
-\Delta w & =(1-m) w^{\alpha}, & & x \in \Omega, t>0, \\
w & =0, & & x \in \partial \Omega,
\end{aligned}
$$

where $\int_{\Omega} w(x) d x=m \in \mathbb{R}$. We test (10) with $x \cdot \nabla u$, integrate over $\Omega$ and use the divergence theorem, to derive the following identity:

Lemma 3 (Pohožaev's Identity). Let $w$ be a classical solution of (10) and $\Omega$ bounded. Then

$$
(1-m)\left(\frac{n}{\alpha+1}-\frac{n-2}{2}\right) \int_{\Omega} w^{\alpha+1}=\frac{1}{2} \int_{\partial \Omega}(x \cdot \nu) w_{\nu}^{2} d \sigma .
$$

Therefore one can derive the following condition for problem (10):

Proposition 4. If $\Omega$ is a star-shaped domain with respect to the origin $0 \in \mathbb{R}^{n}$ (i.e. if $x \cdot \nu>0$ on $\partial \Omega)$ then for

$$
(m-1)\left(\frac{n}{\alpha+1}-\frac{n-2}{2}\right)>0
$$


with $\alpha \geq 1, n>3$, problem (10) does not have any non-negative classical solution. More precisely, problem (10) does not have any non-negative classical solution when:

$$
m_{0}>1 \text { and } \alpha<\frac{n+2}{n-2} \text { or } m_{0}<1 \text { and } \alpha>\frac{n+2}{n-2} .
$$

Remark 5. Although the above non-existence result is for Dirichlet boundary condition, it motivates us to study further the possibility of blow-up for the nonlocal Fisher-KPP (1).

Additionaly, it is still unclear whether blow-up occurs. For example by following Kaplan's method for (1), one can investigate further.

Consider the following auxiliary eigenvalue problem.

$$
\begin{gathered}
\Delta \phi+\mu \phi=0, \quad x \in \Omega, \\
\mathcal{B} \phi \equiv \frac{\partial \phi}{\partial \hat{n}}+\phi=0, \quad x \text { on } \partial \Omega,
\end{gathered}
$$

then we have for an eigenpair $(\mu, \phi)$, such that $\mu, \phi>0$ and $\phi$ bounded. We also take that $\int_{\Omega} \phi(x) d x=1$.

Now we study (1) with the same boundary condition as in (12). We test (1) with the eigenfunction $\phi$, integrate by parts and use problem (12) to derive:

$$
\begin{aligned}
\dot{A}(t) & =\frac{d}{d t} \int_{\Omega} \phi u d x=\int_{\Omega} \phi \Delta u d x+\int_{\Omega} \phi u^{\alpha}(1-m(t)) d x \\
& =-\mu \int_{\Omega} u \phi d x+(1-m(t)) \int_{\Omega} u^{\alpha} \phi d x .
\end{aligned}
$$

At the same time for the mass one can verify that,

$$
m^{\prime}(t)+\int_{\partial \Omega} u d \sigma=(1-m) \int_{\Omega} u^{\alpha} d x
$$

then if we start with initial data such that $1-m(0)>0$, we can see that $m$ will be at most increasing until a time $t_{*} \leq \infty$ when $m\left(t_{*}\right)=1$. For all $t>t_{*}$ we will have $m(t)=1$.

We can apply Jensen's inequality,

$$
\dot{A}(t)=-\mu \int_{\Omega} u \phi d x+(1-m(t)) \int_{\Omega} u^{\alpha} \phi d x \geq-\mu A(t)+C(\Omega)(1-m(t)) A^{\alpha}
$$

which gives us blow-up in finite time $t^{*}$ if $t^{*}<t_{*}$. 


\section{Dynamics of total mass and global well-posedness for small $\alpha$}

In this section we focus on the homogeneous Neumann condition most commonly used in the biological motivation.

The evolution of the total mass plays a key role in our proof for the global existence of the classical solution. Therefore, we firstly give the evolution of mass $\int_{\Omega} u(t) d x$ in time.

Lemma 6. For $m_{0}>0$, the mass $\int_{\Omega} u(t) d x=m(t)$ satisfies

$$
\min \left\{1, m_{0}\right\} \leq m(t) \leq \max \left\{1, m_{0}\right\}
$$

Furthermore, we have the following decay estimates

$$
|1-m(t)| \leq\left|1-m_{0}\right| e^{-\min \left\{1, m_{0}^{\alpha}\right\} t}
$$

Proof. We return to the original problem (1) and integrate it over $\Omega$ to get:

$$
m^{\prime}(t)=(1-m(t)) \int_{\Omega} u^{\alpha} d x
$$

There are two possibilities depending on the initial mass.

- If we start at time $t_{0}$ where $1-m\left(t_{0}\right)>0$, we can see that $m^{\prime}(t)$ is positive and therefore $m$ increases in time. Moreover, with the use of Jensen's inequality we get by using $m(t) \geq m_{0}$,

$$
m^{\prime}(t) \geq(1-m(t)) m^{\alpha}(t) \geq(1-m(t)) m_{0}^{\alpha} .
$$

By solving this inequality we get a lower bound on the speed with which $m(t)$ increases to 1 i.e.

$$
m(t) \geq 1-e^{-m_{0}^{\alpha} t}\left(1-m_{0}\right)
$$

- If $1-m\left(t_{0}\right)<0$, then $m$ decreases in time. By monotonicity, we get $m(t) \leq m_{0}$ and again by Jensen's inequality,

$$
m^{\prime}(t) \leq(1-m(t)) m^{\alpha}(t)<1-m(t)
$$

then we get that

$$
m(t) \leq 1+\left(m_{0}-1\right) e^{-t}
$$

By putting together the above two cases we have the expected results.

Next we focus on the global existence of the classical solution to (1). We will use the following ODE inequality from [6], which was also used in [7]. 
Lemma 7. Assume $y(t) \geq 0$ is a $C^{1}$ function for $t>0$ satisfying

$$
y^{\prime}(t) \leq \alpha-\beta y(t)^{a}
$$

for $a>1, \alpha>0, \beta>0$, then $y(t)$ has the following hyper-contractive property

$$
y(t) \leq(\alpha / \beta)^{1 / a}+\left[\frac{1}{\beta(a-1) t}\right]^{\frac{1}{a-1}} \quad \text { for any } t>0 .
$$

Furthermore, if $y(0)$ is bounded, then

$$
y(t) \leq \max \left(y(0),(\alpha / \beta)^{1 / a}\right) .
$$

The proof of global existence heavily depends on the following two a priori estimates, Proposition 8 and Proposition 9, and then we will use the compactness arguments to close the proof. Due to the preliminary discussion, we will divide the a priori estimates into two cases $m_{0}<1$ and $m_{0} \geq 1$. Firstly, we focus on $m_{0}<1$.

Proposition 8. Let $n \geq 1$ and $m_{0}<1$. If $\alpha$ satisfies

$$
\begin{aligned}
1 \leq \alpha<1+2 / n, & n \geq 3 \\
1 \leq \alpha<2, & n=1,2
\end{aligned}
$$

then for any $1<k<\infty$, the nonnegative solution of (1) satisfies

$$
\|u(\cdot, t)\|_{L^{k}(\Omega)} \leq C\left(m_{0}, k, \alpha\right)+C\left(m_{0}, k, \alpha\right) t^{-\frac{k-1}{\alpha-1}} \quad \text { for any } t>0 .
$$

Moreover, if $u_{0}(x) \in L^{k}(\Omega)$, then

$$
\|u(\cdot, t)\|_{L^{k}(\Omega)} \leq \max \left\{\left\|u_{0}(x)\right\|_{L^{k}(\Omega)}, C\left(m_{0}, k, \alpha\right)\right\},
$$

and for any $0<T<\infty$

$$
\nabla u^{\frac{k}{2}} \in L^{2}\left(0, T ; L^{2}(\Omega)\right)
$$

Proof. Since $m_{0}<1$, by lemma 6 one has $m_{0} \leq m(t) \leq 1$. Using $k u^{k-1}$ as a test function for equation (1) and integrating it by parts

$$
\begin{gathered}
\frac{d}{d t} \int_{\Omega} u^{k} d x=-\frac{4(k-1)}{k} \int_{\Omega}\left|\nabla u^{\frac{k}{2}}\right|^{2} d x+k \int_{\Omega} u^{k+\alpha-1} d x\left(1-\int_{\Omega} u d x\right), \\
\frac{d}{d t} \int_{\Omega} u^{k} d x+\frac{4(k-1)}{k} \int_{\Omega}\left|\nabla u^{\frac{k}{2}}\right|^{2} d x+k m(t) \int_{\Omega} u^{k+\alpha-1} d x=k \int_{\Omega} u^{k+\alpha-1} d x .
\end{gathered}
$$


Choosing $1<k^{\prime}<k+\alpha-1$, combining Hölder's inequality and the Sobolev embedding theorem one has

$$
\begin{aligned}
& \int_{\Omega} u^{k+\alpha-1} d x=\int_{\Omega} u^{\lambda \frac{k}{2} \frac{2(k+\alpha-1)}{k}} u^{(1-\lambda) \frac{k}{2} \frac{2(k+\alpha-1)}{k}} d x \\
\leq & \left\|u^{\frac{k}{2}}\right\|_{L^{p}(\Omega)}^{\lambda \frac{2(k+\alpha-1)}{k}}\left\|u^{\frac{k}{2}}\right\|_{L^{\frac{2 k^{\prime}}{k}}(\Omega)}^{(1-\lambda) \frac{2(k+\alpha-1)}{k}} \\
\leq & C(k)\left(\left\|\nabla u^{\frac{k}{2}}\right\|_{L^{2}(\Omega)}^{\lambda}\left\|u^{\frac{k}{2}}\right\|_{L^{\frac{2 k^{\prime}}{k}}(\Omega)}^{1-\lambda}+\left\|u^{\frac{k}{2}}\right\|_{L^{2}(\Omega)}^{\lambda}\left\|u^{\frac{k}{2}}\right\|_{L^{\frac{2 k^{\prime}}{k}(\Omega)}}^{1-\lambda}\right) \\
\leq & C(k)\left(\left\|\nabla u^{\frac{k}{2}}\right\|_{L^{2}(\Omega)}^{\frac{2 \lambda(k+\alpha-1)}{k}}\left\|u^{\frac{2(k+\alpha-1)}{2}}\right\|_{L^{\frac{2 k^{\prime}}{k}}(\Omega)}^{\frac{2(1-\lambda)(k+\alpha-1)}{k}}+\left\|u^{\frac{k}{2}}\right\|_{L^{2}(\Omega)}^{\frac{2 \lambda(k+\alpha-1)}{k}}\left\|u^{\frac{k}{2}}\right\|_{L^{\frac{2 k^{\prime}}{k}(\Omega)}}^{\frac{2(1-\lambda)(k+\alpha-1)}{k}}\right),
\end{aligned}
$$

where $\lambda$ is the exponent from Hölder's inequality, i.e.

$$
\lambda=\frac{\frac{k}{2 k^{\prime}}-\frac{k}{2(k+\alpha-1)}}{\frac{k}{2 k^{\prime}}-\frac{1}{p}} \in(0,1),
$$

and $p$ satisfies

$$
\begin{cases}p=\frac{2 n}{n-2}, & n \geq 3, \\ \frac{2(k+\alpha-1)}{k}<p<\infty, & n=2, \\ p=\infty, & n=1 .\end{cases}
$$

Now we will divide the analysis into three cases $n \geq 3, n=2$ and $n=1$.

For $n \geq 3, p=\frac{2 n}{n-2}$ and then

$$
\lambda=\frac{\frac{k n}{2 k^{\prime}}-\frac{k n}{2(k+\alpha-1)}}{\frac{k n}{2 k^{\prime}}+1-\frac{n}{2}} \in(0,1),
$$

with $k>\max \left\{\frac{(n-2)(\alpha-1)}{2}, 1\right\}$. Taking $k^{\prime}>\frac{(\alpha-1) n}{2}$, simple computations arrive at

$$
\frac{2 \lambda(k+\alpha-1)}{k}=\frac{\frac{k n}{k^{\prime}}+\frac{(\alpha-1) n}{k^{\prime}}-n}{\frac{k n}{2 k^{\prime}}+1-\frac{n}{2}}<2 .
$$

To sum up, for $k^{\prime}>\max \left\{\frac{(\alpha-1) n}{2}, 1\right\}$, thanks to the Young's inequality, from (21) one has

$$
\begin{aligned}
\int_{\Omega} u^{k+\alpha-1} d x \leq & \frac{k-1}{k^{2}}\left\|\nabla u^{\frac{k}{2}}\right\|_{L^{2}(\Omega)}^{2}+C(k)\left\|u^{\frac{k}{2}}\right\|_{L^{\frac{2 k^{\prime}}{k}}(\Omega)}^{(1-\lambda) \frac{2(k+\alpha-1)}{k} \frac{1}{1-\frac{\lambda(k+\alpha-1)}{k}}} \\
& +C(k)\left\|u^{\frac{k}{2}}\right\|_{L^{2}(\Omega)}^{\frac{2 \lambda(k+\alpha-1)}{k}}\left\|u^{\frac{k}{2}}\right\|_{L^{\frac{2 k^{\prime}}{k}}(\Omega)}^{\frac{2(1-\lambda)(k+\alpha-1)}{k}} .
\end{aligned}
$$


Letting

$$
r=(1-\lambda) \frac{2(k+\alpha-1)}{k} \frac{1}{1-\frac{\lambda(k+\alpha-1)}{k}},
$$

recalling $m(t) \geq m_{0}$, together (20) with (25) we arrive at

$$
\begin{aligned}
& \frac{d}{d t} \int_{\Omega} u^{k} d x+k m_{0} \int_{\Omega} u^{k+\alpha-1} d x+\frac{3(k-1)}{k}\left\|\nabla u^{\frac{k}{2}}\right\|_{L^{2}(\Omega)}^{2} \\
\leq & C(k)\|u\|_{L^{k^{\prime}}(\Omega)}^{\frac{k r}{2}}+C(k)\|u\|_{L^{k}(\Omega)}^{\lambda(k+\alpha-1)}\|u\|_{L^{k^{\prime}}(\Omega)}^{(1-\lambda)(k+\alpha-1)} .
\end{aligned}
$$

On the other hand, using Hölder's inequality with $1<k^{\prime}<k+\alpha-1$ we have

$$
\begin{aligned}
\|u\|_{L^{k^{\prime}}(\Omega)} & \leq C\|u\|_{L^{k+\alpha-1}(\Omega)}^{\theta}\|u\|_{L^{1}(\Omega)}^{1-\theta}, \\
\|u\|_{L^{k}(\Omega)} & \leq C\|u\|_{L^{k+\alpha-1}(\Omega)}^{\eta}\|u\|_{L^{1}(\Omega)}^{1-\eta}
\end{aligned}
$$

where

$$
\theta=\frac{(k+\alpha-1)\left(k^{\prime}-1\right)}{k^{\prime}(k+\alpha-2)} \in(0,1), \quad \eta=\frac{(k+\alpha-1)(k-1)}{k(k+\alpha-2)} \in(0,1) .
$$

Hence

$$
\|u\|_{L^{k^{\prime}}(\Omega)}^{\frac{k r}{2}} \leq\left(C\|u\|_{L^{k+\alpha-1}(\Omega)}^{\theta}\|u\|_{L^{1}(\Omega)}^{1-\theta}\right)^{\frac{k r}{2}} \leq C\left(m_{0}, k\right)\|u\|_{L^{k+\alpha-1}(\Omega)}^{\frac{k r \theta}{2}} .
$$

Taking (27)-(31) into account we obtain that

$$
\begin{aligned}
& \frac{d}{d t} \int_{\Omega} u^{k} d x+k m_{0}\|u\|_{L^{k+\alpha-1}(\Omega)}^{k+\alpha-1}+\frac{3(k-1)}{k}\left\|\nabla u^{\frac{k}{2}}\right\|_{L^{2}(\Omega)}^{2} \\
\leq & C\left(m_{0}, k\right)\|u\|_{L^{k+\alpha-1}(\Omega)}^{\frac{k r \theta}{2}}+C\left(m_{0}, k\right)\|u\|_{L^{k+\alpha-1}(\Omega)}^{(k+\alpha-1)[\lambda \eta+(1-\lambda) \theta]} .
\end{aligned}
$$

Here

$$
\begin{aligned}
\frac{k r}{2} & =(1-\lambda)(k+\alpha-1) \frac{1}{1-\frac{\lambda(k+\alpha-1)}{k}}, \\
\frac{k+\alpha-1}{\theta} & =\frac{k+\alpha-2}{1-\frac{1}{k^{\prime}}} .
\end{aligned}
$$

Recalling the definition of $\theta, \eta, \lambda$, direct computations show that $\lambda \eta+(1-\lambda) \theta<1$. For

$$
1 \leq \alpha<1+\frac{2}{n}
$$

one can derive that

$$
\frac{k r \theta}{2}<k+\alpha-1
$$


Next for $n=2, \frac{2(k+\alpha-1)}{k}<p<\infty$, by proceeding the similar arguments to the case $n \geq 3$ from (24) to (32), we obtain that for

$$
1 \leq \alpha<2-\frac{2}{p}
$$

(34) holds true. When $n=1, p=\infty$, then for $1 \leq \alpha<2$, (34) also holds true.

Therefore, combining the three cases $n \geq 3, n=2$ and $n=1$, using Young's inequality we obtain from (32) that

$$
\begin{aligned}
& \frac{d}{d t} \int_{\Omega} u^{k} d x+k m_{0}\|u\|_{L^{k+\alpha-1}(\Omega)}^{k+\alpha-1}+\frac{3(k-1)}{k}\left\|\nabla u^{\frac{k}{2}}\right\|_{L^{2}(\Omega)}^{2} \\
\leq & \frac{k m_{0}}{4}\|u\|_{L^{k+\alpha-1}(\Omega)}^{k+\alpha-1}+\frac{k m_{0}}{4}\|u\|_{L^{k+\alpha-1}(\Omega)}^{k+\alpha-1}+C\left(m_{0}, k\right) .
\end{aligned}
$$

In addition, Hölder's inequality yields that

$$
\left(\|u\|_{L^{k}(\Omega)}^{k}\right)^{1+\frac{\alpha-1}{k-1}} \leq\|u\|_{L^{k+\alpha-1}(\Omega)}^{k+\alpha-1}\|u\|_{L^{1}(\Omega)}^{\frac{\alpha-1}{k-1}} .
$$

Then using lemma 7, we solve the ODE inequality

$$
\frac{d}{d t} \int_{\Omega} u^{k} d x+\frac{k m_{0}}{2\|u\|_{L^{1}(\Omega)}^{\frac{\alpha-1}{k-1}}}\left(\int_{\Omega} u^{k} d x\right)^{1+\frac{\alpha-1}{k-1}} \leq C\left(m_{0}, k\right)
$$

to obtain that for any $1<k<\infty$,

$$
\|u\|_{L^{k}(\Omega)}^{k} \leq C\left(m_{0}, k, \alpha\right)+\left[\frac{C\left(m_{0}, k, \alpha\right)}{t}\right]^{\frac{k-1}{\alpha-1}} \quad \text { for any } t>0 .
$$

Furthermore, if $u_{0}(x) \in L^{k}(\Omega)$ for any $1<k<\infty$, then taking $y(t)=\int_{\Omega} u^{k} d x$ in lemma 7 one has

$$
\|u\|_{L^{k}(\Omega)} \leq \max \left\{\left\|u_{0}(x)\right\|_{L^{k}(\Omega)}, C\left(m_{0}, k, \alpha\right)\right\} .
$$

Now we integrate (36) from 0 to $T$ in time, then we can obtain that for any $T>0$

$$
\int_{\Omega} u^{k}(T) d x+\int_{0}^{T} \int_{\Omega}\left|\nabla u^{\frac{k}{2}}\right|^{2} d x d t+\int_{0}^{T} \int_{\Omega} u^{k+\alpha-1} d x d t \leq \int_{\Omega} u_{0}^{k} d x+C\left(m_{0}, k\right) T
$$

from which we derive

$$
\nabla u^{\frac{k}{2}} \in L^{2}\left(0, T ; L^{2}(\Omega)\right) \quad \text { for any } T>0
$$

This completes the proof.

For $m_{0} \geq 1$, owing to lemma 6 , we know $m(t) \geq 1$ for any $t>0$, thus we have the following result 
Proposition 9. Let $n \geq 1$ and $\alpha \geq 1$. Assume $u_{0} \in L_{+}^{1}(\Omega)$ and $\int_{\Omega} u_{0}(x) d x=m_{0} \geq 1$, Then for any $1<k<\infty$, the nonnegative solution of (1) satisfies that for any $t>0$

$$
\begin{aligned}
& \|u\|_{L^{k}(\Omega)}^{k} \leq C\left(m_{0}, k\right)+\left[\frac{C\left(m_{0}, k\right)}{t}\right]^{\frac{n(k-1)}{2}}, \quad n \geq 3 \\
& \|u\|_{L^{k}(\Omega)}^{k} \leq C\left(m_{0}, k\right)+\left[\frac{C\left(m_{0}, k\right)}{t}\right]^{k-1}, \quad n=1,2 .
\end{aligned}
$$

Moreover, if $u_{0} \in L^{k}(\Omega)$, then

$$
\int_{0}^{\infty} \int_{\Omega}\left|\nabla u^{\frac{k}{2}}\right|^{2} d x d t \leq \int_{\Omega} u_{0}^{k} d x
$$

Proof. Recalling lemma 6 , we know that if $m_{0}>1$, then $m(t) \geq 1$ for any $t>0$. Hence the $L^{k}$ estimates (20) can be reduced to

$$
\frac{d}{d t} \int_{\Omega} u^{k} d x+\frac{4(k-1)}{k} \int_{\Omega}\left|\nabla u^{\frac{k}{2}}\right|^{2} d x \leq 0 .
$$

For $n \geq 1$, using Hölder's inequality one has that for any $1<k<\infty$, the following estimate holds

$$
\begin{aligned}
\|u\|_{L^{k}(\Omega)}^{k} & \leq\|u\|_{L^{\frac{k p}{2}}(\Omega)}^{k \theta}\|u\|_{L^{1}(\Omega)}^{k(1-\theta)} \\
& =\left\|u^{\frac{k}{2}}\right\|_{L^{p}(\Omega)}^{2 \theta}\|u\|_{L^{1}(\Omega)}^{k(1-\theta)},
\end{aligned}
$$

where $\theta=\frac{k-1}{k-\frac{2}{p}}$ and

$$
\begin{cases}p=\frac{2 n}{n-2}, & n \geq 3 \\ 2<p<\infty, & n=2 \\ p=\infty, & n=1 .\end{cases}
$$

Thanks to the Sobolev embedding theorem and Young's inequality, from (45) one has

$$
\begin{aligned}
\left(\|u\|_{L^{k}(\Omega)}^{k}\right)^{\frac{1}{\theta}} & \leq C(n)\left(\left\|\nabla u^{\frac{k}{2}}\right\|_{L^{2}(\Omega)}^{2}+\left\|u^{\frac{k}{2}}\right\|_{L^{2}(\Omega)}^{2}\right)\|u\|_{L^{1}(\Omega)}^{\frac{k(1-\theta)}{\theta}} \\
& \leq C\left(m_{0}, n\right)\left\|\nabla u^{\frac{k}{2}}\right\|_{L^{2}(\Omega)}^{2}+C\left(m_{0}, n\right)\|u\|_{L^{k}(\Omega)}^{k} \\
& \leq C\left(m_{0}, n\right)\left\|\nabla u^{\frac{k}{2}}\right\|_{L^{2}(\Omega)}^{2}+\frac{1}{2}\left(\|u\|_{L^{k}(\Omega)}^{k}\right)^{\frac{1}{\theta}}+C\left(m_{0}, n, k\right) .
\end{aligned}
$$

Plugging the above estimates into (44) yields that

$$
\frac{d}{d t} \int_{\Omega} u^{k} d x+C\left(m_{0}, n, k\right)\left(\int_{\Omega} u^{k} d x\right)^{\frac{1}{\theta}}+C\left(m_{0}, n, k\right)\left\|\nabla u^{\frac{k}{2}}\right\|_{L^{2}(\Omega)}^{2} \leq C\left(m_{0}, k, n\right),
$$


solving the ODE inequality we have that for any $t>0$

$$
\begin{gathered}
\|u\|_{L^{k}(\Omega)}^{k} \leq C\left(m_{0}, k, n\right)+\left[\frac{C\left(m_{0}, k, n\right)}{t}\right]^{\frac{n(k-1)}{2}}, \quad n \geq 3, \\
\|u\|_{L^{k}(\Omega)}^{k} \leq C\left(m_{0}, k, n\right)+\left[\frac{C\left(m_{0}, k, n\right)}{t}\right]^{k-1}, \quad n=1,2 .
\end{gathered}
$$

Moreover, if $u_{0} \in L^{k}(\Omega)$, then (44) directly yields

$$
\|u\|_{L^{k}(\Omega)} \leq\left\|u_{0}\right\|_{L^{k}(\Omega)} .
$$

Next integrating (44) from 0 to $\infty$ in time we obtain that

$$
\int_{0}^{\infty} \int_{\Omega}\left|\nabla u^{\frac{k}{2}}\right|^{2} d x d t \leq \int_{\Omega} u_{0}^{k} d x
$$

This closes the proof.

Remark 10. In fact, (41) and (42) also hold true for heat equation, and the uniform boundedness in time of the $L^{k}$ norm depends only on the initial mass, not depends on the initial $L^{k}$ norm.

Now we have obtained the necessary a priori estimates to complete the proof of Theorem 1. It can be proved by standard methods and for the convenience of the reader we mention the key steps in the following. First of all, from the above estimates, we can take $k=2$ and $k=2 \alpha$ in Proposition 8 and 9 to get the estimates for $\|\nabla u\|_{L^{2}\left(L^{2}(0, T)\right)}$ and $\left\|u_{t}\right\|_{L^{2}\left(H^{-1}(0, T)\right)}$ for any $T>0$. By Aubin-Lions lemma $[14,32]$, we have the strong compactness of $u$ in $L^{2}$ so that the nonlinear terms can be handled. Therefore, the global existence of weak solutions (in the sense of distributions) can be obtained by standard compactness argument. Secondly, from the estimates of the weak solution in Proposition 8 and Proposition 9, the nonlinear term $u^{\alpha}(1-m(t)) \in L^{k}([0, T] \times \Omega), \forall k>1$ for any $T>0$. The solution is a strong $W_{k}^{2,1}$ solution from classical parabolic theory, [18, 19]. By Sobolev embedding, we can bootstrap it to get that classical solution.

As we can see from the above arguments, equation (1) has classical solutions. Next, we will prove that the solution converges to the solution of heat equation exponentially fast.

Proof of Theorem 2. The difference between the two equations is

$$
(u-v)_{t}+\Delta(u-v)=u^{\alpha}(1-m(t))
$$

Let $\bar{u}(t)=\int_{\Omega} u(x, t) d x$ and $\bar{v}(t)=\int_{\Omega} v(x, t) d x$. By (15), we have $\bar{u}_{t}=m^{\prime}(t)=(1-m(t)) \int_{\Omega} u^{\alpha} d x$. $\bar{v}_{t}(t)=0$ because of $\bar{v}(t)=\bar{v}_{0}$. Therefore,

$$
(u-v)_{t}-(\bar{u}-\bar{v})_{t}+\Delta(u-v)=u^{\alpha}(1-m(t))-(1-m(t)) \int_{\Omega} u^{\alpha} .
$$


The standard $L^{2}$ estimate shows that,

$\frac{1}{2} \frac{d}{d t} \int_{\Omega}|(u-v)-(\bar{u}-\bar{v})|^{2} d x+\int_{\Omega}|\nabla(u-v)|^{2} d x=(1-m(t)) \int_{\Omega}\left[\left(u^{\alpha}-\int_{\Omega} u^{\alpha} d y\right)((u-v)-(\bar{u}-\bar{v}))\right] d x$.

By taking $k=2 \alpha$ in Proposition 8 and Proposition 9, we get

$$
\begin{aligned}
& \frac{1}{2} \frac{d}{d t} \int_{\Omega}|(u-v)-(\bar{u}-\bar{v})|^{2} d x+\int_{\Omega}|\nabla(u-v)|^{2} d x \\
\leq & \frac{1}{2}|1-m(t)| \int_{\Omega}|(u-v)-(\bar{u}-\bar{v})|^{2} d x+C|1-m(t)|,
\end{aligned}
$$

where $C$ depend on $m_{0}, \alpha$ and $\left\|u_{0}\right\|_{L^{2 \alpha}(\Omega)}$. Applying Poincaré inequality and lemma 6 , we get

$$
\begin{aligned}
& \frac{1}{2} \frac{d}{d t} \int_{\Omega}|(u-v)-(\bar{u}-\bar{v})|^{2} d x+C(\Omega) \int_{\Omega}|(u-v)-(\bar{u}-\bar{v})|^{2} d x \\
\leq & C e^{-C t} \int_{\Omega}|(u-v)-(\bar{u}-\bar{v})|^{2} d x+C e^{-C t} .
\end{aligned}
$$

From the above ODE we get the following estimate,

$$
\int_{\Omega}|(u-v)-(\bar{u}-\bar{v})|^{2} d x \leq C_{1} e^{-C_{2} t},
$$

where $C_{1}, C_{2}$ are constants depending on $m_{0}$ and $\left\|u_{0}\right\|_{L^{2 \alpha}(\Omega)}$. Thus, the proof of Theorem 2 is complete.

\section{Acknowledgments}

This work is partially supported from DFG Project CH 955/3-1. Shen Bian is partially supported by National Science Foundation of China (Grant No. 11501025) and the Fundamental Research Funds for the Central Universities (Grant No. ZY1528).

\section{References}

[1] M. Anguiano, P.E. Kloeden, T. Lorenz. Asymptotic behaviour of nonlocal reaction-diffusion equations, Nonlinear Analysis, 73 (2010), 3044-3057.

[2] J. M. Ball, Remarks on blow-up and nonexistence theorems for nonlinear evolution equations, Quart. J. Math. Oxford, (2), 28 (1977), 473-486.

[3] J. M. Ball, Finite time blow-up in nonlinear problems, Nonlinear Evolution Equations (1977), Academic Press, 189-205. 
[4] J. W. Bebernes and A. Bressan, Thermal behaviour for a confined reactive gas, J. Differential Equations, 44 (1982), pp. 118-133.

[5] J. W. Bebernes and R. Ely, Comparison techniques and the method of lines for a parabolic functional equation, Rocky Mountain J. Math., 12 (1982), pp. 723-733.

[6] S. Bian and J.-G. Liu, Dynamic and steady states for multi-dimensional Keller-Segel model with diffusion exponent $m>$ 0, Comm Math Phy., 323 (2013), 1017-1070.

[7] S. Bian, J.-G. Liu and C. Zou, Ultra-contractivity for Keller-Segel model with diffusion exponent $m>1-2 / d$, Kinetic and Related Models, 7(1) (2014), 9-28.

[8] C. Budd, B. Dold, and A. Stewart, Blowup in a partial differential equation with con- served first integral, SIAM J. Appl. Math., 53 (1993), pp. 718-742.

[9] W. Deng, Y. Li, C. Xie. Semilinear reaction-diffusion systems with nonlocal sources, Mathematical and Computer Modelling, 37 (2003), 937-943.

[10] Fisher RA. The wave of advance of advantageous genes, Ann Eugenics 1937, 7, 355-69.

[11] H. Fujita, On the nonlinear equations $\Delta u+e^{u}=0$ and $v_{t}=\Delta v+e^{v}$, Bull. Amer. Math. Soc., 75 (1969), 132-135.

[12] H. Fujita, On some nonexistence and nonuniqueness theorems for nonlinear parabolic equations, Proc. Symp. Pure Math. XVIII, Nonlinear Functional Analysis Amer. Math. Soc., 28 (1970), $105-113$.

[13] H. Fujita, On the blowing up of solutions of the Cauchy problem for $u_{t}=\Delta u+u^{1+\alpha}$, J. Fac. Sci. Univ. Tokyo Sect. IA Math. 13 (1966) 109-124.

[14] Simon, Jacques, Compact sets in the space $L^{p}(O, T ; B)$, Annali di Matematica pura ed applicata 146.1 (1986): 65-96.

[15] B. Hu and Hong-ming Yin, Semilinear parabolic equations with prescribed energy, Rend. Circ. Mat. Palermo, 44 (1995), pp. 479-505.

[16] S. Kaplan, On the growth of solutions of quasilinear parabolic equations, Comm. Pure Appl. Math. 16 (1963), 305-330. 
[17] Kolmogorov AN, Petrovsky IG, Piskunov NS., Investigation of the equation of diffusion combined with increasing of the substance and its application to a biology problem, Bull Moscow State Univ Ser A: Math and Mech 1937;1(6):1-25.

[18] O. A. Ladyzenskaja, V. A. Solonnikov and N. N. Ural'ceva, Linear and Quasilinear Equations of Parabolic Type, Transl. Math. Monog. Vol. 23, Amer. Math. Soc., Providence, R.I., 1968.

[19] G.M. Lieberman, Second Order Parabolic Partial Differential Equations, World Scientific, 1996.

[20] Q. Liu, Y. Chen, S. Lu, Uniform blow-up profiles for nonlinear and nonlocal reaction-diffusion equations, Nonlinear Analysis, 71 (2009), 1572-1583.

[21] Lorz, Alexander and Mirrahimi, Sepideh and Perthame, Benoit, Dirac mass dynamics in multidimensional nonlocal parabolic equations, Comm. Part. Diff. Eq., 2011, 36, 1071-1098.

[22] Lorz, Alexander and Lorenzi, Tommaso and Clairambault, Jean and Escargueil, Alexandre and Perthame, Benoit, Effects of space structure and combination therapies on phenotypic heterogeneity and drug resistance in solid tumors, arXiv:1312.6237v1.

[23] Lorz, Alexander and Lorenzi, Tommaso and Hochberg, Michael E and Clairambault, Jean and Perthame, Benoit, Populational adaptive evolution, chemotherapeutic resistance and multiple anti-cancer therapies, ESAIM: M2AN, 2013, 47, 377-399.

[24] C. V. Pao, Blowing-up of solution for a nonlocal reaction-diffusion problem in combustion theory, J. Math. Anal. Appl., 166 (1992), 591-600.

[25] Pavol Quittner, Philippe Souplet, Superlinear parabolic problems, Blow-up, global existence and steady states, Birkhäuser Advanced Texts, 2007.

[26] P. Souplet, Blow-up in nonlocal reaction-diffusion equations, Siam J. Math. Anal. 29,1301-1334, 1998.

[27] P. Rouchon, Universal bounds for global solutions of a diffusion equation with a nonlocal reaction term, J. Diff. Eq., 193, 75-94, (2003).

[28] V. Volpert. Elliptic partial differential equations. Volume 1. Fredholm theory of elliptic problems in unbounded domains, Birkhäuser, 2011. 
[29] V. Volpert, Elliptic partial differential equations, Vol2 Reaction-diffusion equations, Birkhäuser, 2014.

[30] V. Volpert, S. Petrovskii, Reaction-diffusion waves in biology, Physics of Life Reviews 6, 267310, (2009).

[31] V. Volpert, V. Vougalter, Existence of stationary pulses for nonlocal reaction-diffusion equations, preprint.

[32] Chen, Xiuqing, Ansgar Jüngel, and Jian-Guo Liu, A note on Aubin-Lions-Dubinskii lemmas, Acta Applicandae Mathematicae, 1-11, (2013).

[33] X. Wang, W. Wo, Long time behavior of solutions for a scalar nonlocal reaction-diffusion equation, Arch. Math., 96, 483-490, (2011).

[34] M. Wang and Y. Wang, Properties of positive solutions for non-local reaction-diffusion problems, Math. Methods Appl. Sci., 19, 1141-1156, (1996).

[35] Zeldovich YaB, Frank-Kamenetskii DA, A theory of thermal propagation of flame, Acta Physicochim USSR 9, 341-50, 1938. 